Ersch. in: Computational intelligence in data mining / Giacomo della Riccia, Rudolf Kruse, Hans-Joachim Lenz (eds.). - Wien [u.a.] : Springer, 2000. - S. 111-126. - (International Centre for Mechanical Sciences: Courses and lectures ; 408). - ISBN 3-211-83326-9

\title{
LEARNING FUZZY MODELS AND POTENTIAL OUTLIERS
}

\author{
M.R. Berthold \\ University of California at Berkeley, Berkeley, CA, USA
}

\begin{abstract}
Outliers or distorted attributes very often severely interfere with data analysis algorithms that try to extract few meaningful rules. Most methods to deal with outliers try to completely ignore them. This can be potentially harmful since the very outlier that was ignored might have described a rare but still extremely interesting phenomena. In this paper we describe an approach that tries to build an interpretable model while still maintaining all the information in the data. This is achieved through a two stage process. A first phase builds an outlier-model for data points of low relevance, followed by a second stage which uses this model as filter and generates a simpler model, describing only examples with higher relevance, thus representing a more general concept. The outlier-model on the other hand may point out potential areas of interest to the user. Preliminary experiments indicate that the two models in fact have lower complexity and sometimes even offer superior performance.
\end{abstract}

\section{Introduction}

Many datasets obtained from real-world systems contain distorted elements, for example due to errors in measurements, sensor-failures, or simple recording problems. If the resulting data is to be analyzed by means of extracting an interpretable model from this data, these so-called "outliers" are often difficult to ignore. Many existing methodologies to build models from data will try to incorporate the outliers, making the resulting model more complex and harder to interpret $[4,6,10,11,13]$.

In this paper an approach is discussed which aims to model an evolving set of potential outliers through an additional outlier-model. This results in two models, one representing a more general concept which offers better understandability, and the other concentrating on potential outliers or regions with low evidence in the observed data. The presented approach is based on sets of fuzzy rules as a way to model imprecise relationships $[14,15]$. Based on an existing, fast algorithm that constructs fuzzy rule sets from data [7], the presented

\footnotetext{
* M. Berthold was in part supported by a stipend of the "Gemeinsame Hochschulsonderprogramm III von Bund und Ländern" through the DAAD, BISC, and the DFG.
} 
approach builds a model for the entire data in a first phase. After completion this model is analyzed automatically and the parts with low relevance in respect to the training data are moved to the outlier model. The outlier model is then used as a filter for the second phase. The second phase generates a more general model, representing the data points with higher evidence. The final pair of models consists of one model for potential outliers and one model which represents the more general behavior. The resulting general model is less complex and therefore easier to interpret whereas the outlier-model may point out potential areas of interest to the user.

We will start out by describing a method to build fuzzy models based on example data, followed by a description of the two-stage fuzzy model generation. Thereafter we present results on two datasets, demonstrating the effects of the proposed methodology.

\section{Learning Fuzzy Models from Data}

In the following we will concentrate on $n$-dimensional feature spaces with one dependent variable which is either continuous (for an approximation problem) or denotes a set of classes (in case of a classification task). The goal of the discussed methods is to generate a set of rules which describe some available example data. The used rules describe an implication:

\section{$\mathcal{R}:$ if antecedent then consequent with weight $w$}

and we will focus on the most commonly used form of rules, consisting of an antecedent in form of a constraint on the input variables:

$$
x_{1} \text { is } A_{1} \text { and } \cdots \text { and } x_{n} \text { is } A_{n} \quad \text { or short : } \quad \boldsymbol{x} \text { is } \boldsymbol{A}
$$

where the $A_{i}(1 \leq i \leq n)$ describe fuzzy sets which are defined through their membership functions $\mu_{A_{i}}: \mathbb{R} \rightarrow[0,1]$. The consequent of such rules assigns a certain class $c$ in case of a classifier

\section{$\mathcal{R}:$ if $x$ is $\boldsymbol{A}$ then class $c$}

where $c$ denotes one of $C$ possible classes. In case of a fuzzy graph describing a functional relationship, the consequent specifies a granule for the dependent or output variable

$$
\mathcal{R} \text { : if } x \text { is } A \text { then } y \text { is } B
$$

where $B$ again denotes a fuzzy set, defined through a membership function $\mu_{B}$ : $\mathbb{R} \rightarrow[0,1]$. Of interest is sometimes also a weight parameter $w$ which usually represents the percentage of training patterns explained by this rule. In the following two sections the differences between fuzzy models used for classification and approximation are highlighted before an algorithm to construct both types of models is discussed. 


\subsection{Fuzzy Classifiers}

For a classifier the consequent assigns class $c$ out of a finite number of classes $C$. The degree of membership for a certain pattern $\boldsymbol{x}$ for rule $\mathcal{R}$ is simply computed through the membership degree of the antecedent where the conjunction $\wedge$ is often implemented through the minimum:

$$
\mu_{\mathcal{R}}(\boldsymbol{x})=\mu_{A_{1}}\left(x_{1}\right) \wedge \cdots \wedge \mu_{A_{n}}\left(x_{n}\right)=\min \left\{\mu_{A_{1}}\left(x_{1}\right), \cdots, \mu_{A_{n}}\left(x_{n}\right)\right\}
$$

In this $n$-dimensional feature space the area of influence of each rule $R$ is thus specified by the vector of membership functions $\left(A_{1}, \cdots, A_{n}\right)$. A set of rules is then defined through

$$
\mathbf{R}=\left\{\mathcal{R}^{(r)} \mid 1 \leq r \leq R\right\}
$$

where $R$ indicates the number of rules. Of course, each of these rules only assigns one specific class $c^{(r)}$ out of $\{1, \cdots, C\}$.

The combined degree of membership for a certain class $c$ is computed through a disjunction $\vee$ which is implemented through the maximum:

$$
\mu_{\mathbf{R}}^{c}(\boldsymbol{x})=\bigvee\left\{\mu_{\mathcal{R}^{(r)}}(\boldsymbol{x}) \mid c^{(r)}=c\right\}=\max \left\{\mu_{\mathcal{R}^{(r)}}\left(x_{1}\right) \mid c^{(r)}=c\right\}
$$

In a prediction scenario with equal risk, the class with the highest degree of membership would typically be chosen.

\subsection{Fuzzy Approximators}

For approximation the rule "if $x$ is $\boldsymbol{A}$ then $y$ is $B$ " can also be seen as a constraint on a joint variable $(\boldsymbol{x}, y)$, that is,

$$
(\boldsymbol{x}, y) \text { is } \boldsymbol{A} \times B
$$

where $\times$ denotes the Cartesian product. The membership function of $A \times B$ is again given using $\wedge$ as a conjunction operator:

$$
\mu_{\boldsymbol{A} \times B}(\boldsymbol{x}, y)=\mu_{\boldsymbol{A}}(\boldsymbol{x}) \wedge \mu_{B}(y)=\min \left\{\mu_{\boldsymbol{A}}(\boldsymbol{x}), \mu_{B}(y)\right\}
$$

or, more precisely

$$
\mu_{\boldsymbol{A} \times \boldsymbol{B}}(\boldsymbol{x}, y)=\min \left\{\mu_{A_{1}}\left(x_{1}\right), \cdots, \mu_{A_{n}}\left(x_{n}\right), \mu_{B}(y)\right\}
$$

Zadeh [14] also uses the term fuzzy point to denote such a rule $\boldsymbol{A} \times B$. A collection $\mathbf{R}$ of rules can now be regarded as forming a superposition of $R$ fuzzy points:

$$
(\boldsymbol{x}, y) \text { is }\left(\boldsymbol{A}_{1} \times B_{1}+\cdots+\boldsymbol{A}_{R} \times B_{R}\right)
$$

where + denotes the disjunction operator (usually defined as maximum). Zadeh calls this characterization of a dependency fuzzy graph, because the collection of 


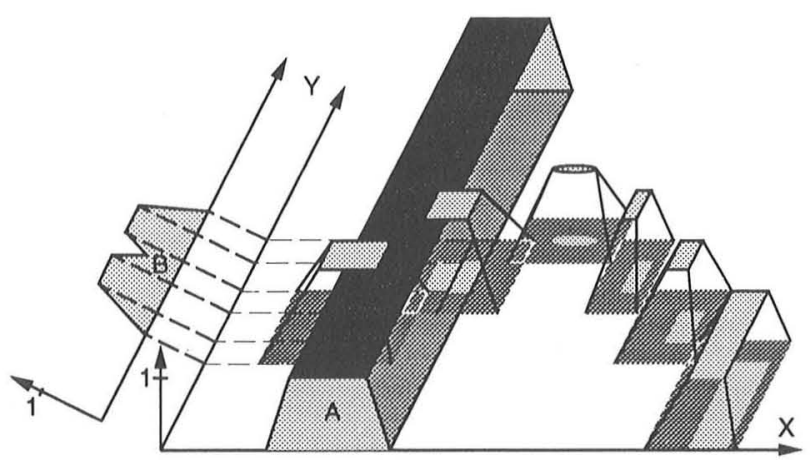

Fig. 1. Interpolation as the intersection of a fuzzy graph with a cylindrical extension of $A$.

rules can be seen as a coarse representation of a functional dependency $f^{*}$ of $y$ on $x$. This fuzzy graph $f^{*}$ can thus be defined as:

$$
f^{*}=\sum_{r=1}^{R}\left(A_{r} \times B_{r}\right)
$$

Note that individual rules do not depend on a common set of membership functions on the input variables. Instead each fuzzy point is described through an individual set of membership functions $\boldsymbol{A}_{r}$ which, especially in high dimensional cases, restrict only few attributes. This makes fuzzy graphs a very compact description, especially compared to the approaches that use a "global grid", defined through only one set of membership functions for each input variable (see for example $[12,6]$ ).

The task of interpolation, that is, deriving a linguistic value $B^{\prime}$ for $y$ given an arbitrary linguistic value $\boldsymbol{A}$ for $\boldsymbol{x}$ and a fuzzy graph $f^{*}$ :

$$
\begin{aligned}
& x \text { is } \boldsymbol{A} \\
& f^{*} \text { is } \sum_{j=1}^{m} A_{j} \times B_{j} \\
& y \text { is } B^{\prime}
\end{aligned}
$$

results in an intersection of the fuzzy graph $f^{*}$ with a cylindrical extension of the input fuzzy set $A$. Figure 1 shows an example. This functional dependency can be computed through:

$$
\begin{aligned}
& \mu_{B^{\prime}}=\mu_{f^{*}(\boldsymbol{A})}(y)=\sup _{\boldsymbol{x}}\left\{\mu_{f^{* *}}(\boldsymbol{x}, y) \wedge \mu_{\boldsymbol{A}}(\boldsymbol{x})\right\} \\
& =\sup _{\boldsymbol{x}}\left\{\left(\mu_{\boldsymbol{A}_{1} \times B_{1}}(\boldsymbol{x}, y) \vee \cdots \vee \mu_{\boldsymbol{A}_{\boldsymbol{r}} \times B_{\boldsymbol{r}}}(\boldsymbol{x}, y)\right) \wedge \mu_{\boldsymbol{A}}(\boldsymbol{x})\right\}
\end{aligned}
$$

If a crisp output is desired the resulting fuzzy set $B^{\prime}$ has to be defuzzified, a common approach here is the "Center of Gravity" method which simply computes 
to:

$$
\tilde{y}=\int_{y} y \cdot \mu_{B^{\prime}}(y) \mathrm{d} y
$$

In the following a strategy to construct such fuzzy rule sets based on example data is described in detail.

\subsection{Learning Fuzzy Rules}

In both cases, that is classification or approximation, the resulting training data consists of an input vector $\boldsymbol{x}$, together with a vector of membership values $\boldsymbol{\mu}^{\text {target }}$ for the desired classes or output granules. The algorithm described here also allows for some - but not necessarily all - features having predefined granulation and is based on two methods to build fuzzy rules and fuzzy graphs [7,2]. As apriori information we therefore have the following:

for the output:

- either the number $C^{\text {out }}$ of classes in case of a classifier, or in case of a continuous output variable its granulation into a set of $C^{\text {out }}$ linguistic values, described through membership functions $\mu_{c}^{\text {out }}: \mathbb{R} \rightarrow[0,1]$, with $1 \leq c \leq$ $C^{\text {out }}$.

This also has implications for the possible consequents of the generated rules. In both cases, there is only a limited choices of possible consequents, either one out of $C^{\text {out }}$ classes or one of $C^{\text {out }}$ granules. In the following we will therefore universally denote a consequent by $g \in\left\{1, \cdots, C^{\text {out }},\right\}$ possibly accompanied by a superscript indicating the rule's index.

and for the inputs:

- a number $n_{\text {granul }}$ of features $x_{i}\left(1 \leq i \leq n_{\text {granul }}\right)$ with a predefined granulation, defined through a set of $C_{i}^{\text {in }}$ linguistic values, described through membership functions $\mu_{i}^{\text {in }, c}: \mathbb{R} \rightarrow[0,1]$, with $1 \leq c \leq C_{i}^{\text {in }}$ and $1 \leq i \leq n_{\text {granul }}$.

- and a number $n_{\text {free }}$ of features $x_{i}\left(n_{\text {granul }}+1 \leq i \leq n=n_{\text {granul }}+n_{\text {free }}\right)$ without a-priori defined granulation ${ }^{1}$.

Example 1. In case of a three-dimensional feature space $(n=3)$ with a continuous output variable "pressure", one could define $C^{\text {out }}=4$ granules, where the linguistic values "very low", "low", "medium", and "high" are defined through the membership functions $\mu_{c}^{\text {out }}(c=1, \ldots, 4)$. The three input features could consist of one granulated feature $\left(n_{\text {granul }}=1\right)$ temperature, with three linguistic values "cold", "warm", and "hot" $\left(C_{1}^{\text {in }}=3\right)$ and another two free features $x_{2}$ and $x_{3}\left(n_{\text {free }}=2\right)$ for two sensor values.

${ }^{1}$ For clarity of representation we assume that the features are ordered with respect to being granulated or not. This does, however, not affect generality of the discussed approach. 
In addition to this information a set of training examples exists:

$$
\mathbf{T}=\left\{\left(\boldsymbol{x}^{(m)}, \boldsymbol{\mu}^{\text {target },(m)}\right) \mid 1 \leq m \leq M\right\}
$$

where $M$ indicates the number of examples in the set $\mathbf{T}$. In case of a classifier the values $\mu_{c}^{\text {target, }(m)}$ indicate the degree of membership to class $c$ for training example $m$. For a continuous output $\mu_{c}^{\text {target, }(m)}$ specifies the desired degree of membership to granule $c\left(1 \leq c \leq C^{\text {out }}\right)$.

The goal of the algorithm is thus to generate a set of rules $\mathbf{R}$, which describe the training data $\mathbf{T}$.

$$
\mathbf{R}=\left\{\mathcal{R}^{(r)} \mid 1 \leq r \leq R\right\}
$$

Each of the $R$ rules is specified through a set of constraints on the input domain and the index of the associated granule or class $g^{(r)}\left(1 \leq g^{(r)} \leq C^{\text {out }}\right)$ :

$$
\mathcal{R}^{(r)}: \text { if } \bigwedge_{i=1}^{n_{\text {granul }}} \operatorname{cond}_{\text {granul }, i}^{(r)} \text { and } \bigwedge_{i=n_{\text {granul }}+1}^{n} \operatorname{cond}_{\text {free }, i}^{(r)} \text { then } g^{(r)} \text { with } w^{(r)}
$$

where the constraint consists of two parts, cond $\mathrm{granul}, i^{(r)}$ and $\operatorname{cond}_{\mathrm{free}, i}^{(r)}$.

- The first part describes the restrictions on the granulated part of the feature space:

$$
\operatorname{cond}_{\mathrm{granul}, i}^{(r)}=x_{i} \text { is } \bigvee_{c=1}^{C_{i}^{\text {in }}}\left(s_{i}^{c} \wedge \mu_{i}^{\text {in }, c}\right)
$$

and $s_{i}^{c}$ specifies the applicable linguistic values for that specific feature ${ }^{2}$. If $s_{i}^{c}=1$, value $c$ is part of the condition, whereas $s_{i}^{c}=0$ indicates its absence in cond $\mathrm{granul}, i^{(r)}$.

Example 2. Assuming a linguistic variable "temperature" (index $i$ ) for rule $r$, the condition cond $\mathrm{granul}, i^{(r)}$ could for example contain two linguistic values "cold" and "warm" out of the set of three possible values "cold" $\left(\mu_{i}^{\text {in, } 1}\right)$, "warm" $\left(\mu_{i}^{\text {in }, 2}\right)$, and "hot" $\left(\mu_{i}^{\text {in }, 3}\right)$. Then $C_{i}^{\text {in }}=3$ and $s_{i}^{1}=1, s_{i}^{2}=1$, and $s_{i}^{3}=0$. Rule $\mathcal{R}^{(r)}$ would thus have the following constraint (among others): $\mathcal{R}^{(r)}$ : if $\cdots$ temperature is (cold or warm) $\cdots$ then $\cdots$

In most cases cond ${ }_{\text {granul, } i}^{(r)}$ will actually contain all linguistic values $\left(s_{i}^{\star}=1\right)$, indicating that this feature is not restricted at all.

- The second part of the constraints cond $\mathrm{free}, i_{i}^{(r)}$, are specified through:

$$
\operatorname{cond}_{\text {free }, i}^{(r)}=\left\{\begin{array}{l}
\text { true } \\
x_{i} \text { is }<a_{i}^{(r)}, b_{i}^{(r)}, c_{i}^{(r)}, d_{i}^{(r)}>
\end{array}\right.
$$

which either specify no constraint at all, or a trapezoidal membership function in case a constraint was derived by the training algorithm.

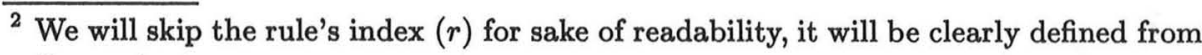
the context. 
The algorithm to derive such a set of rules $\mathbf{R}$ from a set of examples $\mathbf{T}$ is described in the following section.

\subsection{Algorithmic Details}

The training algorithm operates sequentially, considering one example pattern after the other. At start no rules are existent, during training new rules will be introduced and existing rules will be fine tuned. In order to be able to guarantee termination of the algorithm, each rule keeps track of the pattern that originally triggered its creation. This so-called "anchor" is denoted $\lambda^{(r)}$ for rule $\mathcal{R}^{(r)}$. In addition we will use the following abbreviation:

$$
\begin{aligned}
& \operatorname{vol}(\mathcal{R}):=\int \mu_{\mathcal{R}}(\boldsymbol{x}) \mathrm{d} \boldsymbol{x} \\
& =\int \cdots \int \prod_{i=1}^{n_{\text {granul }}}\left(\bigvee_{c=1}^{C_{i}^{\mathrm{in}, \mathrm{c}}} s_{i}^{c} \cdot \mu_{\mathrm{granul}, i}^{\mathrm{in}, c}\left(x_{i}\right)\right) \cdot \prod_{i=n_{\mathrm{granul}}+1}^{n}\left(\mu_{\mathrm{free}, i}^{\mathrm{in}, c}\left(x_{i}\right)\right) \mathrm{d} x_{1} \cdots \mathrm{d} x_{n}
\end{aligned}
$$

which represents the volume rule $\mathcal{R}$ covers in the feature space,

$$
\mu_{\mathcal{R}}(\boldsymbol{x}):=\bigwedge_{i=1}^{n_{\mathrm{granul}}}\left(\bigvee_{c=1}^{C_{i}^{\mathrm{in}, c}} s_{i}^{c} \wedge \mu_{\mathrm{granul}, i}^{\mathrm{in}, c}\left(x_{i}\right)\right) \wedge \bigwedge_{i=n_{\mathrm{granul}}+1}^{n} \mu_{\mathrm{free}, i}^{\mathrm{in}, c}\left(x_{i}\right)
$$

which represents the degree of membership of input $x$ to the antecedent of rule $\mathcal{R}$, that is its degree of fulfillment, and finally

$$
\operatorname{maxindex}(\boldsymbol{\mu}):=\arg \max \left\{\mu_{c} \mid 1 \leq c \leq C^{\text {out }}\right\}
$$

which determines the index of the component in $\mu$ with the maximum degree of membership.

During training for each example pattern $\left(\boldsymbol{x}, \boldsymbol{\mu}^{\text {target }}\right) \in \mathbf{T}$ two different scenario can now occur:

- Covered: in this case a rule index $r_{\text {win }}$ exists which already describes the new pattern, and also has the largest degree of membership among all rules:

$$
\begin{gathered}
\exists r_{\text {win }}: 1 \leq r_{\text {win }} \leq R: g^{\left(r_{\text {win }}\right)}=\operatorname{maxindex}\left(\boldsymbol{\mu}^{\text {target }}\right) \wedge \mu_{\mathcal{R}^{r_{\text {win }}}}(\boldsymbol{x})>0.0 \\
\wedge \forall r^{\prime} \neq r_{\text {win }}: g^{\left(r^{\prime}\right)}=\operatorname{maxindex}\left(\boldsymbol{\mu}^{\text {target }}\right) \rightarrow\left(\mu_{\mathcal{R}^{r_{\text {win }}}}(\boldsymbol{x}) \geq \mu_{\mathcal{R}^{r^{\prime}}}(\boldsymbol{x})\right)
\end{gathered}
$$

The core region of this rule will be increased along the non-granulated features to make sure it covers the new pattern (non-restricted free features do not need to be modified since they cover the pattern anyway):

$$
\begin{aligned}
& \forall i: n_{\text {granul }}+1 \leq i \leq n: \\
& \quad \operatorname{cond}_{\text {free }, i}^{r_{\text {win }}} \neq \text { true } \Rightarrow b_{i}^{\prime r_{\text {win }}}:=\min \left\{b_{i}^{r_{\text {win }}}, x_{i}\right\} \wedge c_{i}^{\prime r_{\text {win }}}:=\max \left\{c_{i}^{r_{\text {win }}}, x_{i}\right\}
\end{aligned}
$$

and the weight of the rule will be incremented, thus keeping track of the number of patterns this rule explains: $w^{\prime r_{\mathrm{win}}}:=w^{r_{\mathrm{win}}}+1$. 
- Commit: in contrast to the above case no rule exists which describes the new pattern. In this case a new rule will be introduced (committed), describing the new example:

$$
\mathcal{R}^{(R+1)}: \text { if true then } g^{(R+1)}:=\operatorname{maxindex}\left(\boldsymbol{\mu}^{\text {target }}\right) \text { with } w^{(R+1)}:=1
$$

Obviously this rule is too general and will need to be specialized subsequently, through adaptation of the antecedent. (Note the weight $w^{(R+1)}$ which is initialized to 1 , indicating that this rule explains one example pattern so far.) We also need to remember the pattern which triggered creation of this rule:

$$
\lambda^{(R+1)}:=\boldsymbol{x} .
$$

For simplicity we also remember the index of the linguistic values of all granulated features which cover the initial pattern $\boldsymbol{x}$ best:

$$
\forall i: 1 \leq i \leq n_{\text {granul }}: c_{\lambda, i}^{(R+1)}:=\arg \max \left\{\mu_{i}^{\text {in }, c}(\boldsymbol{x}) \mid 1 \leq c \leq C_{i}^{\text {in }}\right\}
$$

In addition to the modification and creation of rules describing patterns it is also necessary to actively avoid conflicts, that is, rules describing wrong relations need to be modified. This is done through a third step, called SHRINK, which modifies rules that incorrectly cover a pattern. More precisely, all rules $\mathcal{R}^{(r)}$ will be investigated if they fulfill

$$
1 \leq r \leq R \wedge \mu_{g^{(r)}}^{\text {target }}=0 \rightarrow \mu_{\mathcal{R}(r)}(x)=0
$$

that is, if the rule's consequent has a degree of membership equal to zero for this particular target $\mu^{\text {target }}$ then it also generates a degree of membership equal to zero for the corresponding input vector $\boldsymbol{x}$. For each rule which violates this condition the following SHRINK-procedure will be performed:

- SHRINK: The goal is to specialize the conflicting rule $\mathcal{R}^{(r)}$ in a manner which inhibits the conflict. This is done through specialization of the rule, that is, the area of influence will be shrunk to exclude the new example pattern. To achieve this several alternatives exist, which are applied in the following order:

S1 : Restrict an already constrained granulated feature. In this scenario it is assumed that the conflict can be resolved by tightening an already existing constraint of a granulated feature. Hence we restrict ourselves to:

$$
\begin{aligned}
& \forall i_{\mathbf{S} 1}: 1 \leq i_{\mathbf{S} 1} \leq n_{\mathrm{granul}} \\
& \wedge \exists c: 1 \leq c \leq C_{i_{\mathbf{S} 1}}^{\text {in }} \wedge s_{i_{\mathbf{S} 1}}^{c}=0 \\
& \wedge \exists c: 1 \leq c \leq C_{i_{\mathbf{S} 1}}^{\text {in }} \wedge s_{i_{\mathbf{S 1}}}^{c}=1 \wedge \mu_{i_{\mathbf{S} 1}}^{\text {in }, c}(\boldsymbol{x})=0 \wedge c \neq c_{\lambda, i_{\mathbf{S} 1}}
\end{aligned}
$$

that is, all granulated features for which at least one linguistic value is excluded from cond ${\mathrm{granul}, i_{\mathrm{S} 1}}^{(r)}$ and at least one of the linguistic values 
in cond $_{\text {granul, } i_{\mathbf{S} 1}}^{(r)}$ results in a degree of membership equal to zero but this linguistic value is not the one which covered the anchor of this rule during the initial commit. The last two requirements make sure that we can restrict this feature without completely erasing rule $\mathcal{R}^{(r)}$ or loosing coverage of its anchor. If no such feature can be found, alternative $\mathbf{S 2}$ is probed.

Otherwise we compute the loss in rule $r$ 's volume, assuming that feature $i_{\mathrm{S} 1}$ is used to eliminate the conflict, resulting in a revised rule $\mathcal{R}_{i \mathrm{~S} 1}^{\prime(r)}$ which is similar to rule $\mathcal{R}^{(r)}$, the only difference being the constraint on the granulated feature $i_{\mathbf{S} 1}$ :

$$
\operatorname{cond}_{\mathrm{granul}, i_{\mathbf{S} 1}}^{(r)}:=x_{i_{\mathbf{S} 1}} \text { is } \bigvee_{c=1}^{C_{i_{\mathbf{S} 1}}^{\mathrm{n}}} \underbrace{\left(s_{i_{\mathbf{S} 1}}^{c} \wedge\left(\mu_{i_{\mathbf{S 1}}}^{\mathrm{in}, c}(\boldsymbol{x})=0\right)\right)}_{=: s_{\mathbf{i} 1}^{\prime c}} \mu_{i_{\mathbf{S} 1}^{\mathrm{in}, c}}
$$

all other constraints remain unchanged. From there we can compute the respective loss in rule $r$ 's volume:

$$
\operatorname{loss}_{i_{\mathrm{S} 1}}=\operatorname{vol}\left(\mathcal{R}^{(r)}\right)-\operatorname{vol}\left(\mathcal{R}_{i_{\mathrm{S} 1}}^{\prime(r)}\right)
$$

We then choose the one feature $i_{\mathbf{S 1} \text {, best }}$ which minimizes this loss in volume, thus keeping the restricted rule as large as possible:

$$
i_{\mathbf{S} 1, \text { best }}=\arg \min \left\{\operatorname{loss}_{i_{\mathbf{S} 1}}\right\}
$$

and replace rule $\mathcal{R}^{(\nabla)}$ in the new set of rules:

$$
\mathbf{R}^{\prime}:=\mathbf{R} \cup\left\{\mathcal{R}_{i \mathrm{~s} 1, \text { best }}^{\prime(r)}\right\} \backslash\left\{\mathcal{R}^{(r)}\right\} .
$$

Example 3. A rule $\mathcal{R}$ describes the fact that one buys oil when the temperature is either "cold" or "warm":

\section{$\mathcal{R}:$ if temperature is cold or warm then buy oil}

This rule might result in a conflict because an example household did not buy oil during "warm" temperatures. The revised rule would then be (through further restriction of feature temperature):

$$
\mathcal{R}^{\prime} \text { : if temperature is cold then buy oil }
$$

$\mathbf{S} 2$ : Restrict an unconstrained granulated feature. If $\mathbf{S} \mathbf{1}$ could not be applied we will try to resolve the conflict through restricting a previously unconstrained, granulated feature. We are therefore looking at the following features:

$$
\begin{aligned}
& \forall i_{\mathbf{S 2}}: 1 \leq i_{\mathbf{S 2}} \leq n_{\text {granul }} \\
& \wedge \forall c: 1 \leq c \leq C_{i_{\mathbf{S 2}}}^{\text {in }} \wedge s_{i_{\mathbf{S 2}}^{c}}^{c}=1 \\
& \wedge \exists c: 1 \leq c \leq C_{i_{\mathbf{S 2}}}^{\text {in }} \wedge \mu_{i_{\mathbf{S 2}}}^{\text {in,c }}(\boldsymbol{x})=0 \wedge c \neq c_{\lambda, i_{\mathbf{S} 2}}
\end{aligned}
$$


that is, all linguistic are contained in the constraint and we again make also sure that at least one linguistic value will remain in the modified constraint and we do not loose coverage of the anchor of this rule. Similar to above, if no such feature can be found, alternative S3 is probed.

Otherwise we proceed analogous to scenario $\mathbf{S 1}$, that is, we compute the possible losses in volume and choose the one feature for shrinkage which results in the minimum loss in volume.

Example 4. The feature "temperature" of the rule from above can now not be restricted any further without eliminating the rule entirely.

\section{$\mathcal{R}$ : if temperature is cold then buy oil}

Therefore the previously unconstrained feature "oil price" will be restricted and $\mathcal{R}$ might become:

\section{$\mathcal{R}^{\prime}$ : if temperature is cold and oilprice is low then buy oil}

S3 : Restrict an already constrained free feature. If none of the granulated features can be restricted to avoid the conflict, one of the remaining free features needs to be used. We will first try to tighten an already existing constraint on a free feature, that is we restrict ourselves to:

$$
\begin{aligned}
& \forall i_{\mathbf{S} \mathbf{3}}: n_{\text {free }}+1 \leq i_{\mathbf{S} \mathbf{3}} \leq n \\
& \wedge \operatorname{cond}_{\text {free }, i_{\mathbf{S} \mathbf{s}}}^{(r)}=x_{i_{\mathbf{S} \mathbf{3}}} \text { is }<a_{i_{\mathbf{S} \mathbf{s}}}^{(r)}, b_{i_{\mathbf{S} \mathbf{s}}}^{(r)}, c_{i_{\mathbf{S} \mathbf{s}}}^{(r)}, d_{i_{\mathbf{S} \mathbf{3}}}^{(r)}>
\end{aligned}
$$

that is, all free features $i$ for which a constraining trapezoidal membership function is already defined. If no such feature can be found, alternative $\mathbf{S} 4$ is probed.

Otherwise - similar to cases $\mathbf{S 1}$ and $\mathbf{S 2}$ - we determine which of these features results in a minimum loss of rule-coverage. First for each feature $i_{\text {SS }}$ we compute a modified trapezoid which eliminates the conflict:

$$
\begin{aligned}
& <a_{i_{\mathbf{S s}}}^{\prime(r)}, b_{i_{\mathbf{S s}}}^{\prime(r)}, c_{i_{\mathbf{S s}}}^{\prime(r)}, d_{i_{\mathbf{S s}}}^{\prime(r)}>
\end{aligned}
$$

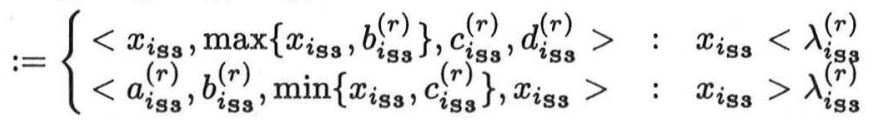

that is, depending on the position of the conflict with respect to the anchor $\lambda_{i_{\mathrm{Ss}}}$ the left or right side of the trapezoid is modified. This ensures that the anchor will always remain inside this rule but the conflict will be moved to the border of the support area.

Similar to $\mathbf{S} 1$ we replace cond $\mathrm{free}_{\text {, } i_{\mathbf{S} 3}}^{(r)}$ using the new trapezoid and compute the loss in volume for the modified rule. One feature $i_{\mathbf{S 3} \text {, best }}$ will then be selected which minimizes this loss and the corresponding rule will be replaced in the set of rules $\mathbf{R}$.

Example 5.

$$
\mathcal{R} \text { : if temperature is cold and } x_{42} \text { is }<2,3,7,9>\cdots
$$


might become, through restriction of feature $x_{42}$ to avoid a conflict at $x_{42}=8$ :

$$
\mathcal{R}^{\prime} \text { : if temperature is cold and } x_{42} \text { is }\langle 2,3,7,8>\cdots
$$

S4 : Restrict an unconstrained free feature. If no constrained free feature can be found, one of the unconstrained free feature needs to be constrained. That is we restrict ourselves to:

$$
\begin{gathered}
\forall i_{\mathbf{S} 4}: n_{\text {free }}+1 \leq i_{\mathbf{S} 4} \leq n \\
\wedge \operatorname{cond}_{\text {free }, i_{\mathbf{S A}}}^{(r)}=\text { true }
\end{gathered}
$$

that is, all free features $i_{\mathbf{S} \mathbf{4}}$ for which a constraining trapezoidal membership function has not been defined. If no such feature can be found, a serious conflict has been encountered, that is, two example points have the same input vector $\boldsymbol{x}$ but conflicting targets $\boldsymbol{\mu}$. Usually this will result in a feedback to the user, pointing out this inconsistency in the data set. Otherwise - similar to case S3 - we determine which of these features results in a minimum loss of rule-coverage. First for each feature $i_{\mathbf{S} 4}$ we compute a new trapezoid which eliminates the conflict:

$$
\begin{aligned}
& <a_{i_{\mathrm{SA}}}^{\prime(r)}, b_{i_{\mathrm{SA}}}^{\prime(r)}, c_{i_{\mathrm{SA}}}^{\prime(r)}, d_{i_{\mathrm{S} 4}^{\prime}}^{\prime(r)}>
\end{aligned}
$$

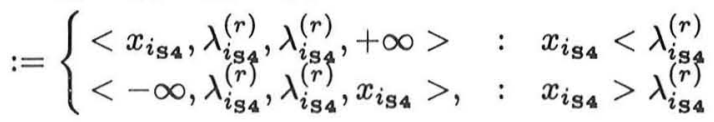

that is, depending on the position of the conflict with respect to the anchor $\lambda_{i_{\mathrm{S}}}$ the left or right side of the trapezoid is restricted. This ensures that the anchor will always remain inside this rule but the conflict $x_{i}$ will be moved to the border of the support area. The core itself is set equal to the anchor $\lambda_{\mathbf{S 4}}$ and will later be enlarged if this rule covers other patterns.

Similar to S3 we then replace $\operatorname{cond}_{\text {free, }, \text { is }}^{(r)}$ and compute the loss in volume for the modified rule. Feature $i_{\mathbf{S 4} \text {,best }}$ will then be selected to minimize this loss and the respective rule will be replaced in the set of rule $\mathbf{R}$.

Example 6.

$$
\mathcal{R}: \text { if temperature is cold } \ldots
$$

might become (through restriction of the previously unconstrained feature $x_{42}$ ):

$$
\mathcal{R}^{\prime} \text { : if temperature is cold and } x_{42} \text { is }\langle-\infty, 3,3,7>\cdots
$$

if $\lambda_{42}=3$, and the conflict occurred at $x_{42}=7$. 


\section{Two Stage Fuzzy Models}

Most existing algorithms to construct fuzzy rule sets from data have tremendous problems with noisy data or data containing outliers. Usually an excessive number of rules is being introduced simply to model noise and/or outliers. This also applies to the algorithm described in the previous section. This is due to the fact that these algorithms aim to generate conflict free rules, that is, examples encountered during training will result in a degree of membership $>0$ only for those rules of the correct class (or granule). Unfortunately in case of spares outliers such an approach will, especially in high-dimensional feature spaces, result in an enormous amount of rules to avoid these conflicts. Figure 2 demonstrates this effect.

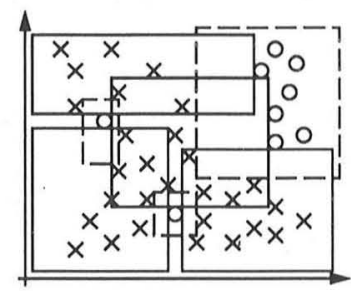

Fig. 2. An example how two outliers (o) produce a high number of rules for the competing class $\times$.

Using the already existing model we can, however, in many cases easily determine parts that have low relevance, based on their weight or another parameter which denotes individual relevance. To measure a rule's relevance often the weight parameter $w^{(r)}$ is used which represents the number of training patterns covered by rule $r$. From this a measure for the importance or relevance of each rule can be derived, by simply using the percentage of patterns covered by this rule:

$$
\Phi\left(\mathcal{R}^{(r)}\right)=\frac{w^{(r)}}{|\mathbf{T}|} .
$$

Other measures which are also used determine the loss of information if rule $r$ is omitted:

$$
\Phi\left(\mathcal{R}^{(r)}\right)=I(\mathbf{R})-I\left(\mathbf{R} \backslash\left\{\mathcal{R}^{(r)}\right\}\right)
$$

where $I(\cdot)$ indicates a function measuring the information content of a rule set. Most commonly used are (a more extensive overview can be found in [3] and also [1]):

- the Gini-index:

$$
I_{\text {Gini }}(\mathbf{R})=1-\sum_{i=1}^{C^{\text {out }}} V_{i}(\mathbf{R})^{2}
$$


- and the fuzzy entropy function:

$$
I_{\text {Entropy }}(\mathbf{R})=-\sum_{i=1}^{C^{\text {out }}}\left(V_{i}(\mathbf{R}) \log _{2} V_{i}(\mathbf{R})\right)
$$

where $V_{i}(\mathbf{R})$ indicates the volume of all rules $\mathcal{R}^{(r)}$ in $\mathbf{R}$ which are assigned to granule $i=g^{(r)}$ :

$$
V_{i}(\mathbf{R})=\int_{\boldsymbol{x}} \max _{\mathcal{R}^{(r)} \in \mathbf{R} \wedge g^{(r)}=i}\left\{\mu^{i}(\boldsymbol{x})\right\} \mathrm{d} \boldsymbol{x}
$$

(In [9] it is shown how this volume can be computed efficiently based on a system of fuzzy rules.)

The choice of relevance-measure is made depending on the nature of the underlying rule generation algorithm, as well as the focus of analysis, i.e. the interpretation of important vs. unimportant or useless data points. Using such a measure of (notably subjective) relevance, we can now extract rules with low relevance from this model, assuming that they describe points in the data which are outliers or spares points:

$$
\mathbf{R}_{\text {outlier }}=\mathbf{R} \backslash\left\{\mathcal{R} \in \mathbf{R}: \Phi(\mathcal{R})<\theta_{\text {outlier }}\right\}
$$

Using this "outlier"-model as filter for a second training phase will then generate a new fuzzy model which has less rules with higher significance. In fact, the original training data $\mathbf{T}$ is filtered and only data points which are not covered by the outlier model will be used to construct the new model:

$$
\mathbf{T}_{\text {clean }}=\left\{\left(\boldsymbol{x}, \boldsymbol{\mu}^{\text {target }}\right) \in \mathbf{T} \mid \forall \mathcal{R} \in \mathbf{R}_{\text {outlier }}: \mu_{\mathcal{R}}(\boldsymbol{x}) \leq \theta_{\text {filter }}\right\}
$$

Figure 3 shows the flow of this procedure. Note, how the initial model is being used to extract the outlier-model. This model is then in turn used as a filter for the existing training data to generate the final model. In Figure 4 (left) the outlier model is shown, covering the two single points of class 0 . Now the

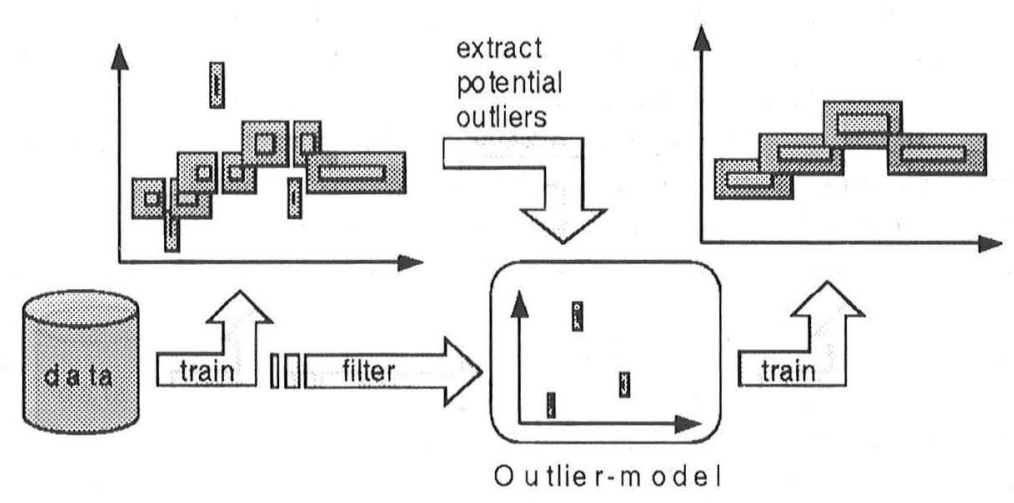

Fig. 3. The role of the two models during training. 


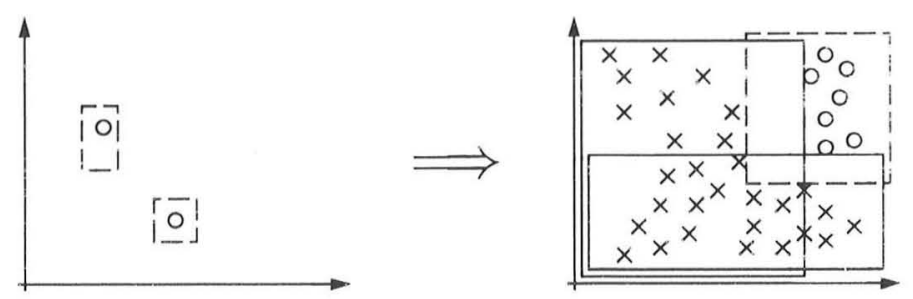

Fig. 4. The same example, but here the additional outlier-model (left) reduces the number of generated rules in the second stage model (right).

second phase can ignore these points and generate two large rules describing the remaining data points of class $\times$ (right). In the following section we will show how this effects the size of the rule sets on two real-world datasets.

\section{Experiments}

Experiments on two datasets from the Statlog-archive [8] were performed to demonstrate the effect of the proposed methodology. The relevance function $\Phi\left(R^{(j)}\right)=w^{(j)}$ with a threshold of $\theta=5$ was used, that is rules which cover less then five patterns were considered irrelevant.

The first dataset contains images from Satellites (Satimage-dataset), patterus with 36 attributes have to be separated into 6 different classes. All together 4435 training and 2000 test patterns were used. Table 1 (left) shows the results. Here "Standard" stands for the normal algorithm which generates fuzzy rules in one run. $\mathcal{H}_{1}$ indicates the general model generated through the algorithm explained above. The number of rules for both models is shown in the last column. The number before the brackets indicates the size of the rule-set of the general model $\mathcal{H}_{1}$, whereas the number in brackets denotes the number of rules of the outlier model $\mathcal{H}_{0}$. It is interesting to see how already without any additional distortion $(0.0 \%)$ the two-stage model shows slightly better performance using a considerable smaller number of rules (270. vs. 393). Note also how the error rate on the unseen test data increases much slower with increases in distortion for the two-stage model. The gap between the sizes of the two models widens as well.

The second dataset is the Segment data from the same archive. Here 19 inputs and 7 classes are used with 2079 training and 220 test patterus. Table 1 (right) shows the results on this dataset. Here the effect in performance is not as obvious. Still noticeable, however, is the difference in model size. While the size of the separate outlier-model increases with increasing distortion, the size of the model representing the more general behavior grows much slower. 
Table 1. Results on the Satimage (left) and Segment (right) dataset

\begin{tabular}{|c||c|c|c|}
\hline $\begin{array}{c}\text { level of } \\
\text { distort. }\end{array}$ & $\begin{array}{c}\text { used } \\
\text { model }\end{array}$ & $\begin{array}{c}\text { error on } \\
\text { test data }\end{array}$ & \#rules \\
\hline $0.0 \%$ & Stand. & $15.9 \%$ & 393 \\
& $\mathcal{H}_{1}\left(\mathcal{H}_{0}\right)$ & $13.5 \%$ & $270(60)$ \\
\hline $1.0 \%$ & Stand. & $17.1 \%$ & 394 \\
& $\mathcal{H}_{1}\left(\mathcal{H}_{0}\right)$ & $13.5 \%$ & $313(81)$ \\
\hline $2.0 \%$ & Stand. & $18.1 \%$ & 404 \\
& $\mathcal{H}_{1}\left(\mathcal{H}_{0}\right)$ & $12.9 \%$ & $295(109)$ \\
\hline $5.0 \%$ & Stand. & $18.1 \%$ & 479 \\
& $\mathcal{H}_{1}\left(\mathcal{H}_{0}\right)$ & $12.4 \%$ & $334(145)$ \\
\hline $10.0 \%$ & Stand. & $22.3 \%$ & 578 \\
& $\mathcal{H}_{1}\left(\mathcal{H}_{0}\right)$ & $15.2 \%$ & $379(199)$ \\
\hline
\end{tabular}

\begin{tabular}{|c||c|c|c|}
\hline $\begin{array}{c}\text { level of } \\
\text { distort. }\end{array}$ & $\begin{array}{c}\text { used } \\
\text { model }\end{array}$ & $\begin{array}{c}\text { error on } \\
\text { test data }\end{array}$ & \#rules \\
\hline $0.0 \%$ & Stand. & $3.5 \%$ & 96 \\
& $\mathcal{H}_{1}\left(\mathcal{H}_{0}\right)$ & $3.0 \%$ & $80(12)$ \\
\hline $1.0 \%$ & Stand. & $5.2 \%$ & 108 \\
& $\mathcal{H}_{1}\left(\mathcal{H}_{0}\right)$ & $4.3 \%$ & $86(22)$ \\
\hline $2.0 \%$ & Stand. & $6.9 \%$ & 113 \\
& $\mathcal{H}_{1}\left(\mathcal{H}_{0}\right)$ & $5.6 \%$ & $83(30)$ \\
\hline $5.0 \%$ & Stand. & $6.1 \%$ & 144 \\
& $\mathcal{H}_{1}\left(\mathcal{H}_{0}\right)$ & $3.8 \%$ & $107(37)$ \\
\hline $10.0 \%$ & Stand. & $6.5 \%$ & 151 \\
& $\mathcal{H}_{1}\left(\mathcal{H}_{0}\right)$ & $6.5 \%$ & $106(45)$ \\
\hline
\end{tabular}

\section{Conclusions}

In this paper we discussed a strategy to model potential outliers through an additional outlier-model. This results in two models, one representing a more general concept which offers better understandability, and the other concentrating on potential outliers or regions with low evidence in the observed data. In the future an entire hierarchy of fuzzy models seems to be a promising approach to model large amounts of data and enable the user to investigate the underlying behavior at various levels of granularity, following Lotfi Zadeh's concept of "information granulation".

\section{References}

1. C. Apte, S. Hong, J. Hosking, J. Lepre, E. Pednault, and B. K. Rosen. Decomposition of heterogeneous classification problems. Intelligent Data Analysis, 2(2), 1998. (http://www.elsevier.nl/locate/ida).

2. M. R. Berthold and K.-P. Huber. Constructing fuzzy graphs from examples. Intelligent Data Analysis, 3(1), 1999. (http://www.elsevier.nl/locate/ida).

3. V. Cherkassky and F. Mulier. Learning from Data. John Wiley and Sons Inc., 1998.

4. S. K. Halamuge and M. Glesner. FuNe Deluxe: A group of fuzzy-neural methods for complex data analysis problems. In Proceedings of the EUFIT'95, Aug. 1995.

5. D. Hand, J. Kok, and M. Berthold, editors. Advances in Intelligent Data Analysis. LNCS. Springer Verlag, 1999.

6. C. M. Higgins and R. M. Goodman. Learning fuzzy rule-based neural networks for control. In Advances in Neural Information Processing Systems, 5, pages 350-357, California, 1993. Morgan Kaufmann.

7. K.-P. Huber and M. R. Berthold. Building precise classifiers with automatic rule extraction. In IEEE International Conference on Neural Networks, 3, pages 12631268, 1995.

8. D. Michie, D. J. Spiegelhalter, and C. C. Taylor, editors. Machine Learning, Neural and Statistical Classification. Ellis Horwood Limited, 1994. 
9. R. Silipo and M. R. Berthold. Discriminative power of input features. In [5], 1999.

10. P. K. Simpson. Fuzzy min-max neural networks - part 1: Classification. IEEE Transactions on Neural Networks, 3(5):776-786, Sept. 1992.

11. P. K. Simpson. Fuzzy min-max neural networks - part 2: Clustering. IEEE Transactions on Fuzzy Systems, 1(1):32-45, Jan. 1993.

12. L.-X. Wang and J. M. Mendel. Generating rules by learning from examples. In International Symposium on Intelligent Control, pages 263-268. IEEE, 1991.

13. L.-X. Wang and J. M. Mendel. Generating fuzzy rules by learning from examples. IEEE Transactions on Systems, Man, and Cybernetics, 22(6):1313-1427, 1992.

14. L. A. Zadeh. Soft computing and fuzzy logic. IEEE Software, pages 48-56, Nov. 1994.

15. L. A. Zadeh. Fuzzy logic and the calculi of fuzzy rules and fuzzy graphs: A precis. Multi. Val. Logic, 1:1-38, 1996. 